$$
H^{n}(x)=\frac{R_{n}(x)}{(x-1)^{n}} .
$$

The relation (7) gives many generalizations of (9). For example we can take $m=p^{e}$ in lieu of $m=p$. Further details I hope to give in another paper on Bernoulli numbers and Euler polynomials.

UNIVERSITY OF TEXAS

\title{
A THEOREM ON MEAN RULED SURFACES
}

\section{BY MALCOLM FOSTER}

Consider the ruled surface formed by the normals to a surface $S$ along some curve $C$ on $S$. We ask: What are the curves $C$ for which the line of striction of the ruled surface is the locus of the centers of mean curvature corresponding to $C$ ?

On $S$ we take the lines of curvature parametric. Referred to the moving trihedral of $S$, the direction-cosines of the normal are $(0,0,1)$, and the variations in these are given by*

$$
d X=q d u, \quad d Y=-p_{1} d v, \quad d Z=0 .
$$

Now the displacement of the central point on each generator of the ruled surface is orthogonal both to the normal and to its neighboring position. Hence we have

$$
\delta z=0, \quad q d u \delta x-p_{1} d v \delta y+\delta z=0,
$$

which reduce to

$$
q d u(\xi d u+z q d u)-p_{1} d v\left(\eta_{1} d v-z p_{1} d v\right)=0 .
$$

If in (1) we assign a value to the ratio $d v / d u$, this equation will determine the distance $z$ to the line of striction on the ruled surface defined by this ratio; and if to $z$ we assign a given value, equation (1) will determine the curves, (though not necessarily real), for which this assigned value of $z$ is the distance to the lines of striction.

From (1) we have for the problem at hand,

\footnotetext{
* Eisenhart, Differential Geometry of Curves and Surfaces, pp. 166-174.
} 


$$
z=\frac{p_{1} \eta_{1} d v^{2}-q \xi d u^{2}}{p_{1}^{2} d v^{2}+q^{2} d u^{2}}=\frac{q \eta_{1}-p_{1} \xi}{2 p_{1} q}
$$

which after some simplification may be written

$$
\left(q \eta_{1}+p_{1} \xi\right)\left(p_{1}^{2} d v^{2}-q^{2} d u^{2}\right)=0 .
$$

It is readily seen that the vanishing of the first factor in (2) would mean that $S$ is either a sphere or a plane. Hence, excluding these cases, the equation of the curves defining the mean ruled surfaces of the congruence of normals to $S$ is given by*

$$
q^{2} d u^{2}-p_{1}^{2} d v^{2}=0 .
$$

Since this is identical with the equation which defines the principal surfaces of the Ribaucour congruence for which $S$ is the director surface, we have the following theorem.

THEOREM. The mean ruled surfaces of the congruence of normals to a surface $S$ are represented on $S$ by curves which define also the principal surfaces of the Ribaucour congruence for which $S$ is the director surface.

\section{WESLEYAN UNIVERSITY}

* Foster, Rectilinear congruences referred to special surfaces, Annals of Mathematics, (2), vol. 25 (1923), p. 177. 